\title{
Prevalence of schizophrenia in a refuge for homeless men: a five year follow-up
}

\author{
MAReE Teesson, Research Officer, Inner City Mental Health Service, Sydney, Australia \\ (correspondence: Ms M. Teesson, Clinical Research Unit for Anxiety Disorders, \\ St Vincent's Hospital, 299 Forbes Street, Darlinghurst, NSW 2010, Australia); and \\ NeIL BuHRICH, Staff Specialist in Psychiatry, St Vincent's Hospital, and Clinical \\ Coordinator, Inner City Mental Health Service, Sydney, New South Wales
}

Until the mid 1950s, the management of persons suffering from chronic mental illness in Australia was the responsibility of the large mental hospital. With the advent of psychotropic drugs and concern about the 'negative' influence of the institutional environment on patients there was a shift to shorter but more frequent periods of hospital admission. As the pattern of shorter admissions increased, community services for the mentally ill were expanded in the early 1970s. Between 1950 and 1985 in the state of New South Wales, the numbers of patients in large mental hospitals decreased from 256 to 55 per 100000 population. However, no special arrangements for accommodation, as distinct from treatment, were made for these ex-mental hospital patients. The decline in hospital numbers can be attributed to a general reduction in the length of hospital stays. Initially, there seemed to be no pressing need for extra accommodation in the community as the provision of social security benefits was adequate to allow patients without homes to afford basic accommodation, a situation different to that in the United States (Lamb, 1984).

In the 1980s low cost accommodation became scarce due to increasing economic pressure, especially within the large city centres of Australia. Homelessness among the mentally ill developed into a political issue, and following a government enquiry (Richmond, 1983) it was recommended that community services for the psychiatrically disabled be expanded to include community accommodation for many of the long-stay patients still residing in psychiatric hospitals.

Despite the expanded community services, mental health workers continued to report a growing number of homeless mentally ill people on the streets of Sydney. Similar reports concerning the increasing numbers of homeless mentally ill were reported in the United States and Britain. In 1983 a random sample of men residing in a large refuge in the inner city of Sydney were screened for mental illness (Doutney et al, 1985). The point prevalence of schizophrenia among the residents was found to be between $14 \%$ and $16 \%$. The present study replicates that study by ascertaining the point prevalence of schizophrenia in the same refuge five years later and then explores data which may bear on the reasons for homelessness. Through replication we hope to counter some of the methodological problems which render hazardous generalisations across studies of schizophrenia among the homeless (Susser et al, 1988).

Homelessness among the mentally ill may be a consequence of something more than mere hospital discharge (Lamb, 1984). It is clear that it can be associated with deinstitutionalisation in the sense that three decades ago most of the chronic mentally ill would have been housed in mental hospitals. However, the mentally ill homeless only became highly visible in the 1970s after the absolute decline in hospital census had already leveled off. Thus, the contribution of deinstitutionalisation to homelessness cannot be understood as a simple period effect (Susser et al, 1989). Other important intervening variables which may lead to homelessness must be explored such as the way deinstitutionalisation was implemented, the availability of low cost accommodation and aspects of the schizophrenic process itself. The second aim of this study was to clarify the relationship between availability of accommodation and admission to psychiatric hospital with regard to the schizophrenic men residing at this inner city refuge.

\section{The study}

The refuge is the largest and cheapest shelter for homeless men in the inner city of Sydney. It is a modern building with a capacity to shelter 400-450 men in dormitories of $50-100$ beds. A one in four sample (116) of residents was randomly selected by bed number. The names of the selected men were announced over a public address system and those who responded were asked to complete an interview. Demographic information was collected and the 
Diagnostic Interview Schedule (DIS; Robins et al, 1981) was administered. The DIS is a highly structured psychiatric interview for use by trained lay interviewers, from which major diagnostic categories can be derived using DSM-III criteria.

Any subject who reported psychotic symptoms, had been admitted to a psychiatric hospital or was receiving psychotropic medication was referred to the second author, a psychiatrist with 16 years experience, for a full psychiatric assessment. In order that the psychiatrist did not assume that every subject referred was psychotic, and as a check on the DIS, one in four of the subjects considered not psychotic by the DIS were also referred for the second interview. The psychiatrist categorised the subjects as either not schizophrenic, probably schizophrenic or definitely schizophrenic. The criteria for diagnosis by the psychiatrist were those set out in DSM-III and thus were the same criteria used in the 1983 study. In general, the subjects categorised as probably schizophrenic were probable in the sense that they did not offer adequate information for a DSM-III diagnosis of schizophrenia. All eight metropolitan psychiatric hospitals were approached for records of admissions.

\section{Findings}

One hundred and sixteen bed numbers were selected for the study; $84(72 \%)$ of the occupants agreed to be interviewed, $9(8 \%)$ refused and $23(20 \%)$ did not respond when their names were called. In the 1983 sample 118 subjects were selected and $91(77 \%)$ were interviewed; three (3\%) refused and $24(20 \%)$ did not respond when their names were called.

Of the 84 occupants who agreed to be interviewed, $64(76 \%)$ completed the DIS. Ten gave adequate information for a DSM-III diagnosis of schizophrenia to be made using the DIS; four men gave symptoms consistent with a DSM-III diagnosis of schizophrenia but not adequate information for a diagnosis by the DIS alone; one man who completed the DIS failed to report age of onset of illness and a further five men denied any psychiatric symptoms on the DIS and three refused the interview although all admitted to past psychiatric admissions or current antipsychotic medication. All of these men were referred to the psychiatrist for a second open-ended assessment, as were one in four of the remaining 61 men interviewed. Of the 39 men interviewed by the psychiatrist, 18 were considered on the basis of interview and hospital record definitely to suffer from schizophrenia and four were considered to be probably schizophrenic. This is a revision of our preliminary estimates which were based only on interview. None of the men considered not psychotic at DIS and referred for a second interview were diagnosed as schizophrenic by the psychiatrist. One man was diag- nosed as schizophrenic by the DIS and not by the psychiatrist. He was aged 65 and had been admitted to hospital for the first time in 1979 with a diagnosis of Korsakoff's syndrome. His bizarre confabulations as recorded by the DIS mistakenly allowed a diagnosis of schizophrenia.

The four men diagnosed as probably schizophrenic by the psychiatrist had all been treated with fluphenazine decanoate. If these four men are excluded the prevalence of schizophrenia is $21 \%$; if included, the prevalence rises to $26 \%$. Using the latter figure, the $95 \%$ range of prevalence of schizophrenia expected from the present data is 15-36\%. The 95\% range of prevalence of schizophrenia expected from the 1983 sample was $8-25 \%$. The change in prevalence from $1983(16 \%)$ to $1988(26 \%)$ was not statistically significant.

Of the 18 men diagnosed by the psychiatrist as definitely having schizophrenia, 16 had previous hospital admissions for schizophrenia. The other two had been seen by psychiatrists elsewhere, one had been diagnosed as schizophrenic and the other as an "acute psychotic". Of the four men diagnosed by the psychiatrist as probably having schizophrenia, two had been admitted to hospital for alchoholism but not for schizophrenia, one for "possible schizophreniform psychosis" and one for "personality disorder, dependent type".

Among the remaining 62 men not diagnosed as schizophrenic at interview, $26(42 \%)$ had been admitted to a psychiatric hospital, two with a diagnosis of schizophrenia, but according to the case notes the diagnosis in these two men was equivocal. Among the 32 men who either refused interview or who did not respond when called, four had been admitted to one of the eight psychiatric hospitals and given a diagnosis of schizophrenia.

The median length of stay at the refuge for the 22 men diagnosed as having definite or probable schizophrenia was 1.5 years (mean length of stay was 2.6 years), the shortest stay was two days and the longest ten years. Ten of the 22 schizophrenic men had moved to the refuge from private rental accommodation, seven had moved from a boarding house, one from a hospital and three from their parental home. The mean age of the men diagnosed as suffering from schizophrenia was 46 years $(s . d .=12)$ and of the men judged not to suffer from the disorder the mean age was 54 years $(s . d .=12)$. This difference is not statistically significant. In the 1983 study the age differences were similar, the mean age of the schizophrenic men being 43 years and that of the other men 52 years.

Figure 1 displays the extent of hospital admissions among the 22 subjects who had definite or probable schizophrenia. The mean number of admissions per patient was 12 (range 0 to 38) and the average length of stay was 63 days (range 1 to 2242 days). 


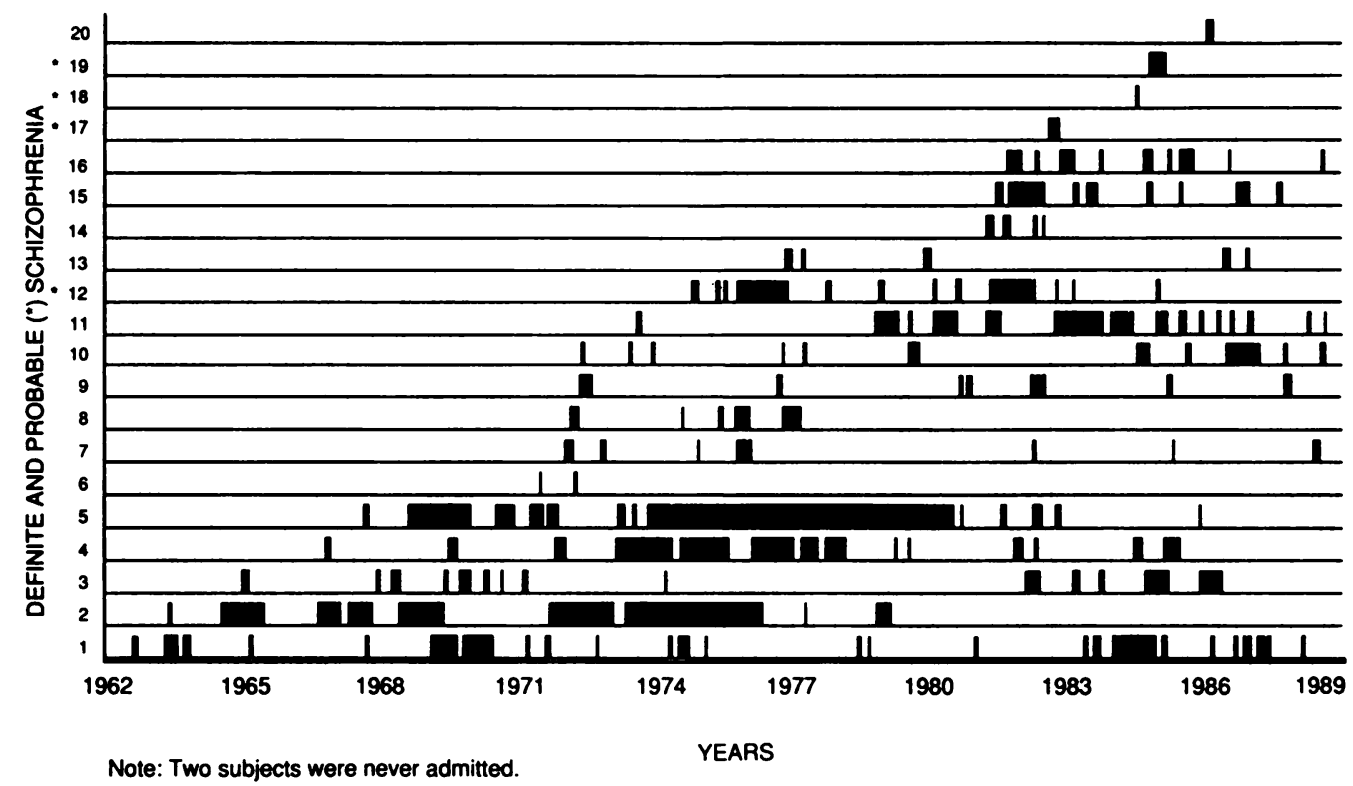

FIG. 1. Psychiatric hospital admissions for the 22 men diagnosed by the psychiatrist as having definite or probable schizophrenia.

\section{Comment}

The mental hospital population' in NSW has been reduced by two thirds over the last three decades. Despite community services, mental health workers continue to report increasing numbers of mentally ill among the homeless (Arce et al, 1983). The present study is the first replication concerning the prevalence of schizophrenia in a refuge for the homeless. In 1983 the prevalence of schizophrenia in this refuge, the largest in Australia, was 14-16\%. Five years later the prevalence of schizophrenia was found to be still unacceptably high at $21-26 \%$. Therefore, on any one night in this 450 bed refuge there are between 70 and 100 men suffering from schizophrenia.

Deinstitutionalisation has been blamed for the high numbers of homeless mentally ill. Inspection of hospital admission records for the present cohort shows that only three of the 22 schizophrenic residents had had a prolonged admission to a psychiatric hospital. Admissions for the remaining 19 were brief and frequent, but the duration of stay in hospital had not altered significantly over the years. In a separate study Andrews et al (1990) found that not one of the 208 long-stay mental patients discharged into supported accommodation in New South Wales had drifted to a refuge for the homeless at 21-month follow-up. Thus, our subjects had not been long-stay hospital patients over the previous three decades, nor had they drifted to the refuge from supported accommodation.
Why, then, is it that we may now be finding increasing numbers of mentally ill among the homeless? Before moving to the refuge the schizophrenic men had largely resided in boarding houses and private accommodation. In Sydney, especially in the inner city, there has been a rapid influx of tourists and, as a result, low cost accommodation has become increasingly scarce. For example, in Sydney (population 3.5 million), the number of local council registered boarding houses fell by $27 \%$ between 1983 and 1988 and in the three months before the present study the average rent of a one bedroom flat had increased by $21 \%$.

It is our view that people with schizophrenia who once could afford a room or boarding house can now no longer do so and must seek shelter in the refuges. In fact, it was only after 1981 when low cost accommodation became scarce in Sydney that 20 of the 22 schizophrenic men began to use the refuge on a regular basis. Clearly low cost accommodation of a poor grade is not all that is necessary to replace hospital facilities and reduce homelessness among the mentally ill. Lamb (1984) has emphasised that what is required is a range of accommodation including low cost housing.

Although the high cost of available accommodation is a key reason for these schizophrenic men presenting at the refuge, other factors may be important in their lack of success in leaving it. Wing \& Brown (1970) argue that persons who have spent a considerable time in institutions develop a lack of 
initiative, apathy, submissiveness to authority, and excessive dependence on the institution. Low standard social conditions may exacerbate symptomatology, particularly negative symptoms (Wing \& Brown, 1970). The combination of the schizophrenic process and poor quality of social environment may contribute to the accumulating numbers of mentally ill in the refuges and shelters for the homeless. Our data support the contention of Lamb (1984) that homelessness among the chronic mentally ill is not simply a result of deinstitutionalisation.

Keogel et al (1988), using an earlier version of the DIS, reported that the prevalence of schizophrenia in skid-row dwellers of Los Angeles was $13.7 \%$. They considered this to be an underestimate as information compiled from other sources, including previous admittance to hospital, suggested the prevalence to be closer to $20 \%$. It is clear from our study that prevalence rates based only on the DIS would have underestimated the prevalence of schizophrenia in the refuge. The main problem in utilising the DIS in such a cohort of men is that approximately one third will fail to complete the interview. However, when the subjects do complete the DIS interview, the correlation between the DIS diagnosis and the psychiatrist's diagnosis is reasonable. As a greater number of studies are using the DIS it is important to note that with a cohort of homeless men the prevalence rate of schizophrenia will be under-reported by at least one third.

\section{Acknowledgement}

The authors thank the staff and guests of the Matthew Talbot Hostel for their co-operation.

\section{References}

Andrews, G., Teesson, M., Stewart, G. \& Hoult, J. (1990) Community placement of the chronic mentally ill. Hospital and Community Psychiatry, 41, 184-188.

Arce, A. A., TADlock, M., Vergare, M. J. \& Shapiro, H. (1983) A psychiatric profile of street people admitted to an emergency shelter. Hospital and Community Psychiatry, 34, 812-817.

DOUTney, C. P., Buhrich, N., Virgona, A., Cohen, A. \& DANIELS, A. (1985) The prevalence of schizophrenia in a refuge for homeless men. Australian and New Zealand Journal of Psychiatry, 19, 233-238.

KeOGel, P., BURnham, A. \& FARR, R. K. (1988) The prevalence of specific psychiatric disorders among the homeless individuals in the inner city of Los Angeles. Archives of General Psychiatry, 45, 1085-1092.

LAMB, H. R. (1984) Deinstitutionalisation and the homeless mentally ill. Hospital and Community Psychiatry, 35, 899-907.

RichMond, D. (1983) The Richmond Report. New South Wales Inquiry into Health Services for Psychiatrically III and the Developmentally Disabled. Sydney: New South Wales Department of Health.

Robins, L. N., Helzer, J. E., Croughan, J. \& Ratcliff, K. S. (1981) National Institute of Mental Health Diagnostic Interview Schedule: its history, characteristics and validity. Archives of General Psychiatry, 38, 381-389.

SUSSER, E., LOVELl, A. \& CONOVER, S. (1988) Unravelling the causes of homelessness and of its association with mental illness. In Epidemiology and the Prevention of Mental Disorders (eds. B. Cooper \& T. Helgason). London: Routledge \& Kegan.

, Conover, S. \& Struening, E. (1989) Problems of epidemiologic method in assessing the type and extent of mental illness among homeless adults. Hospital and Community Psychiatry, 40, 261-265.

WING, J. K. \& BROWN, G. W. (1970) Institutionalism and Schizophrenia. New York: Cambridge University Press.

A full list of references is available on request from $\mathrm{Dr}$ Teesson. 